Jacinta J. Maas

Michael R. Pinsky

Bart F. Geerts

Rob B. de Wilde

Jos R. Jansen

\section{Estimation of mean systemic filling pressure in postoperative cardiac surgery patients with three methods}

Received: 30 November 2011

Accepted: 15 April 2012

Published online: 15 May 2012

(C) The Author(s) 2012. This article is published with open access at Springerlink.com

\begin{abstract}
Purpose: To assess the level of agreement between different bedside estimates of effective circulating blood volume-mean systemic filling pressure (Pmsf), arm equilibrium pressure (Parm) and model analog (Pmsa) - in ICU patients. Methods: Eleven mechanically ventilated postoperative cardiac surgery patients were studied. Sequential measures were made in the supine position, rotating the bed to a $30^{\circ}$ head-up tilt and after fluid loading (500 ml colloid). During each condition four inspiratory hold maneuvers were done to determine Pmsf; arm stop-flow was created by inflating a cuff around the upper arm for $30 \mathrm{~s}$ to measure Parm, and Pmsa was estimated from a Guytonian model of the systemic circulation. Results: Mean Pmsf, Parm and Pmsa across all three states were $20.9 \pm 5.6,19.8 \pm 5.7$ and $14.9 \pm 4.0 \mathrm{mmHg}$, respectively. Bland-Altman analysis for the
\end{abstract}

difference between Parm and Pmsf showed a non-significant bias of $-1.0 \pm 3.08 \mathrm{mmHg}(p=0.062)$, a coefficient of variation $(\mathrm{COV})$ of $15 \%$, and limits of agreement (LOA) of -7.3 and $5.2 \mathrm{mmHg}$. For the difference between Pmsf and Pmsa we found a bias of $-6.0 \pm 3.1 \mathrm{mmHg}$ $(p<0.001)$, COV $17 \%$ and LOA -12.4 and $0.3 \mathrm{mmHg}$. Changes in Pmsf and Parm and in Pmsf and Pmsa were directionally concordant in response to head-up tilt and volume loading. Conclusions: Parm and Pmsf are interchangeable in mechanically ventilated postoperative cardiac surgery patients. Changes in effective circulatory volume are tracked well by changes in Parm and Pmsa.

Keywords Guyton - Venous return . Mean systemic filling pressure . Cardiac output - Circulation

\section{Introduction}

Accurate assessment of the cardiovascular state in the critically ill is difficult because easily measured parameters, such as blood pressure and cardiac output (CO), can coexist with different levels of ventricular pump function and effective circulating blood volume. Thus, identifying the appropriate therapy and targeting specific measurable endpoints of therapy are problematic. Although assessing dynamic changes in arterial pulse pressure or left ventricular stroke volume during ventilation and passive leg- raising maneuvers improves identification of fluid responsiveness, they do not quantify the effective circulating blood volume, or the cause or lack thereof. Although fluid resuscitation therapy is important in the management of unstable patients, excessive fluid resuscitation can be harmful in acute lung injury [1], head injury [2] and postoperative patients [3]. Thus, a measure of effective volume status is useful to avoid volume overload since even volume-overloaded patients may remain volume responsive.

Mean systemic filling pressure (Pmsf) is a functional measure of the effective intravascular volume status. It is 
the pressure anywhere in the circulation during circulatory arrest [4]. Importantly, the central venous pressure (Pcv) to Pmsf pressure difference defines the driving pressure for venous return, and together with the resistance to venous return defines $\mathrm{CO}$. We have shown that Pmsf can be measured in ventilator-dependent patients using inspiratory-hold maneuvers defining Pcv-CO data pairs that when extrapolated to zero $\mathrm{CO}$ reports Pmsf [5, 6]. This calculated Pmsf parameter accurately follows changes in intravascular volume $[5,7]$.

Unfortunately, this inspiratory-hold technique requires a sedated and ventilated patient, not universally seen in critically ill patients. We thus studied two simpler bedside methods for determining Pmsf as previously suggested by Anderson [8] and Parkin [9]. Anderson hypothesized that the circulation of the arm behaves similarly to total systemic circulation during steady state conditions. Accordingly, we measured transient stop-flow forearm arterial and venous equilibrium pressure, referred to as arm equilibrium pressure (Parm). Parkin [9] proposed estimating the effective circulatory volume based on an electrical analog simplification of Guytonian circulatory physiology estimating the mean circulatory pressure (Pmsa) from directly measured Pcv, mean arterial pressure and $\mathrm{CO}$. The aim of our study was to compare the level of agreement among simultaneously measured Pmsf, Parm and Pmsa in three intravascular volume states in critically ill patients.

\section{Materials and methods}

The study was approved by the hospital ethics committee of Leiden University Medical Center (P01.111, 29 January 2002) and carried out in Leiden. Written informed consent was obtained from all patients prior to surgery. The institutional review board of the University of Pittsburgh approved the review and analysis of data. Eleven patients were enrolled and studied after cardiac surgery.

\section{Patients}

We limited our study to cardiac surgery patients requiring pulmonary artery and radial artery catheters for perioperative monitoring. Our study partially used hemodynamic data from the same patients reported in another study, but examined different protocol-based measures [7]. All patients had coronary artery or valvular disease with preserved ventricular function $(\mathrm{EFlv}>0.4)$. Patients with aortic aneurysm, severe peripheral vascular disease, postoperative arrhythmia, postoperative valvular insufficiency or needing artificial pacing or the use of a cardiac assist device were excluded. All subjects were studied during their initial postoperative period in the ICU while sedated (propofol $3.0 \mathrm{mg} \mathrm{kg}^{-1} \mathrm{~h}^{-1}$ and sufentanil $\left.0.06-0.19 \mu \mathrm{g} \mathrm{kg}^{-1} \mathrm{~h}^{-1}\right)$ and mechanically ventilated with airway pressure release ventilation adjusted to achieve normocapnia, with 7-11 ml kg ${ }^{-1}$ tidal volumes, $5 \mathrm{cmH}_{2} \mathrm{O}$ positive end-expiratory pressure, $\mathrm{FiO}_{2} \quad 0.4$ and $f=11-13 \mathrm{~min}^{-1}$ (Evita 4, Dräger AG, Lübeck, Germany). During the study interval all subjects were hemodynamically stable and no changes were made in their vasoactive drug therapy.

\section{Measurements}

All subjects also had a central venous catheter. Arterial pressure $(\mathrm{Pa})$ and $\mathrm{Pcv}$ were recorded on a computer for off-line analysis. Pa and Pcv pressure transducers were referenced to the intersection of the anterior axillar line and the 5th intercostal space, and re-referenced after a $30^{\circ}$ head-up rotation. Airway pressure (Paw) was measured at the proximal end of the endotracheal tube. Beat-to-beat cardiac output $(\mathrm{CO})$ was obtained by Modelflow pulse contour analysis as previously described by us [10-12]. We calibrated the pulse contour CO measurements with three thermodilution $\mathrm{CO}$ measurements equally spread over the ventilatory cycle [11].

We have previously described the inspiratory-hold method for estimating Pmsf [5]. Briefly, four 12-s inspiratory holds were applied at Paw of 5, 15, 25 and $35 \mathrm{cmH}_{2} \mathrm{O}$, respectively. The resulting Pcv and $\mathrm{CO}$ were measured during the plateau phase (between 7 and $12 \mathrm{~s}$ of each inspiratory hold maneuver), and the zero CO intercept of the Pcv and CO pairs estimated Pmsf.

Parm estimates of Pmsf [8] assumes Pa and Pv equilibrium following rapid vascular occlusion. We performed a pilot study in nine patients after either cardiac surgery or cardiopulmonary resuscitation to determine the stop-flow time. We measured arterial and venous pressures in the same hand and created upper extremity blood stop-flow using a rapid cuff inflator (Hokanson E20, Bellevue, Washington) to pressures $50 \mathrm{mmHg}$ above systolic pressure and held occlusion for 35-60 s (Fig. 1). Measurements were performed three times to assess repeatability (Table 1). Arterial and venous pressures equilibrated after 25-30 s of stop-flow, with a mean difference of $-0.73 \pm 1.07 \mathrm{mmHg}$ at $30 \mathrm{~s}$. Thus, we chose the 30-s value of the arterial pressure for Parm for the present study.

The Pmsa estimate [9] uses a mathematical model of the systemic circulation comprising compliant arterial and venous compartments and resistances to blood flow. The model parameters are adjusted to match those of the patient's current measured variables, such that Pmsa $=a \times \mathrm{Pcv}+b \times \mathrm{Pa}+c \times \mathrm{CO}$, where $a$ and $b$ are dimensionless constants $(a+b=1$, typically $a=0.96, b=0.04$ ), and $\mathrm{c}$ has the dimensions of resistance and is a function of the patient's height, weight and age. 


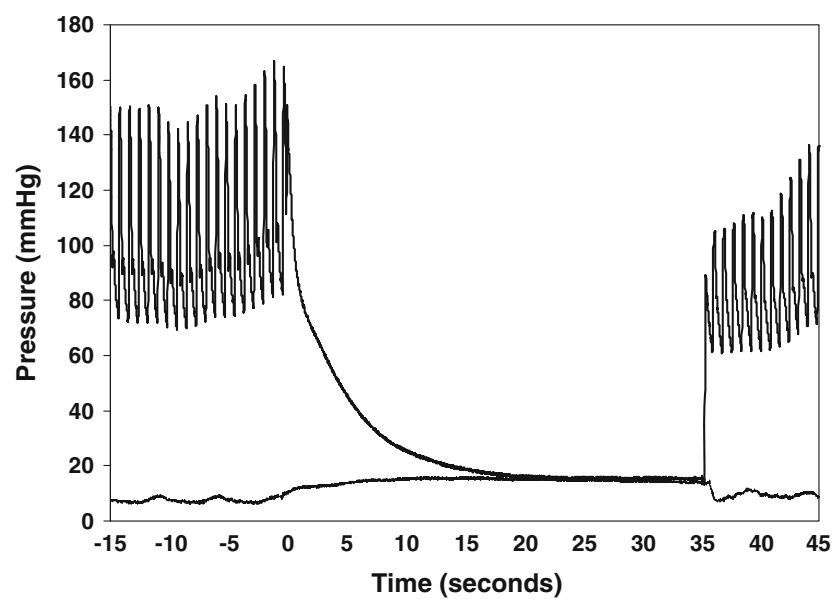

Fig. 1 Representative radial artery pressure and venous pressure trends before ( -15 to $0 \mathrm{~s}$ ), during ( 0 to $35 \mathrm{~s}$ ) and after the occlusion of the upper arm of a patient. Arm vascular occlusion equilibrium pressure (Parm) is taken as the arterial pressure $30 \mathrm{~s}$ after stop-flow. Note the influence of mechanical ventilation on arterial and venous pressure before and after occlusion

$$
\begin{aligned}
& c=0.038 \times(94.17+0.193 \times \text { age }) /\left(4.5 \times\left[0.9^{(\text {age }-15)}\right] \times\right. \\
& \left.0.007184 \times\left[\text { height }^{0.725}\right] \times\left[\text { weight }^{0.425}\right]\right) .
\end{aligned}
$$

\section{Protocol}

Measurements were carried out within $2 \mathrm{~h}$ of arrival in the ICU following initial hemodynamic stabilization. To induce changes in volume status, measurements were performed in the supine position (baseline), in a $30^{\circ}$ headup tilt (HUT) and again in the supine position after $500 \mathrm{ml}$ hydroxyethyl starch (HES 130/0.4) rapid fluid administration (VOL). Measurements of $\mathrm{Pa}, \mathrm{Pv}, \mathrm{Pcv}$ and $\mathrm{CO}$ were done during baseline in the supine position, 2 min after the change to HUT and 2-5 min after fluid loading, with Pmsf, Parm and Pmsa calculated for each step. Repeatability of Parm was determined by two measurements at baseline and after VOL. The study protocol lasted about $60 \mathrm{~min}$. All patients completed all steps of the protocol, and there were no adverse events.
Statistical analysis

After confirming normal distribution of data with the Kolmogorov-Smirnov test, differences among Pmsf, Parm and Pmsa during baseline, HUT and VOL were analyzed using paired $t$ tests. Calculations of bias, precision and limits of agreement (LOA) between Pmsf and both Parm and Pmsa were performed using Bland-Altman analysis with bias reflecting the mean difference between Pmsf and either Parm or Pmsa and precision as the standard deviation (SD) of these differences. After adjustment for the number of observations $(n=33)$, LOA were defined as bias $\pm 2.04 \times$ SD. For repeatability of Parm $(n=40)$ LOAs were bias $\pm 2.02 \times$ SD. The coefficient of variation (COV) was calculated as $100 \% \times \mathrm{SD} /$ mean. Repeatability of Parm was calculated by Bland-Altman analysis using duplicate measurements at baseline and after VOL, which were pooled together. A $p$ value $<0.05$ was considered statistically significant. Unless otherwise stated, data are presented as mean $\pm \mathrm{SD}$.

\section{Results}

Patient characteristics are presented in Table 2 and mean hemodynamic data for the protocol in Table 3. Mean $\mathrm{Pa}$ decreased during HUT and was unchanged with VOL. Pcv, CO, Pmsf, Parm and Pmsa decreased during HUT and increased with VOL.

Pmsf, Parm and Pmsa decreased in all patients during HUT $(3.4 \pm 2.6, \quad 3.0 \pm 2.0$ and $3.7 \pm 2.3 \mathrm{mmHg}$, $p<0.001, p=0.001$, respectively). VOL was associated with an increase in Pmsf, Parm and Pmsa $(8.7 \pm 5.3$, $8.7 \pm 3.8$ and $4.5 \pm 2.1 \mathrm{mmHg}, p<0.001$ all, respectively). Parm was not different from the Pmsf during the baseline, HUT or VOL $(p=0.236, p=0.423$ and $p=0.173$, respectively). However, Pmsf and Pmsa differed significantly for the three conditions $(p<0.001$ all). Pmsf regressed significantly with Parm (Fig. 2a) [slope $=0.944$, correlation coefficient $(R)=0.847]$ and Pmsa (Fig. 2b) (slope $=0.704, R=0.822$ ).

\begin{tabular}{|c|c|c|c|c|c|c|c|c|c|}
\hline \multirow[t]{2}{*}{ Time (s) } & \multicolumn{3}{|l|}{$\mathrm{Pa}$} & \multicolumn{3}{|l|}{$\mathrm{Pv}$} & \multicolumn{3}{|l|}{$\mathrm{Pa}-\mathrm{Pv}$} \\
\hline & $\begin{array}{l}\text { Mean } \\
(\mathrm{mmHg})\end{array}$ & $\begin{array}{l}\text { SD } \\
(\mathrm{mmHg})\end{array}$ & $\begin{array}{l}\text { Repeat } \\
(\%)\end{array}$ & $\begin{array}{l}\text { Mean } \\
(\mathrm{mmHg})\end{array}$ & $\begin{array}{l}\text { SD } \\
(\mathrm{mmHg})\end{array}$ & $\begin{array}{l}\text { Repeat } \\
(\%)\end{array}$ & $\begin{array}{l}\text { Mean } \\
(\mathrm{mmHg})\end{array}$ & $\begin{array}{l}\text { SD } \\
(\mathrm{mmHg})\end{array}$ & $\begin{array}{l}\text { Repeat } \\
(\%)\end{array}$ \\
\hline 15 & 23.32 & 2.41 & 5.45 & 21.96 & 2.05 & 9.20 & 1.35 & 2.69 & 4.89 \\
\hline 20 & 22.11 & 1.88 & 6.11 & 22.12 & 2.02 & 9.58 & -0.01 & 1.62 & 5.52 \\
\hline 25 & 21.42 & 1.56 & 6.91 & 22.06 & 1.91 & 9.79 & -0.63 & 1.02 & 5.18 \\
\hline 30 & 21.08 & 1.38 & 6.55 & 21.81 & 2.05 & 9.58 & -0.73 & 1.07 & 4.55 \\
\hline
\end{tabular}

Table 1 Pilot study arm equilibrium pressure

Effect of time on radial arterial pressure $(\mathrm{Pa})$, peripheral venous pressure $(\mathrm{Pv})$ and the difference between $\mathrm{Pa}$ and $\mathrm{Pv}$ during upper arm stop-flow. The results of a pilot study in nine patients are indicated. Repeat, the averaged repeatability of three sequential measurements $S D$ Standard deviation 
Table 2 Patient characteristics

\begin{tabular}{|c|c|c|}
\hline & Mean & Range \\
\hline Age (years) & 64 & $50-80$ \\
\hline Gender & 9 male, 2 female & \\
\hline Weight (kg) & 86 & $73-112$ \\
\hline Length $(\mathrm{cm})$ & 174 & $158-190$ \\
\hline \multicolumn{3}{|l|}{ Surgery } \\
\hline $\mathrm{CABG}$ & 9 & \\
\hline AVR & 2 & \\
\hline Respiratory rate $\left(\min ^{-1}\right)$ & 12 & $11-13$ \\
\hline $\begin{array}{l}\text { Tidal volume/predicted } \\
\left(\mathrm{ml} \mathrm{kg}^{-1}\right)\end{array}$ & 9 & $7-11$ \\
\hline \multirow[t]{2}{*}{ PEEP $\left(\mathrm{cmH}_{2} \mathrm{O}\right)$} & 5 & \\
\hline & $\begin{array}{l}\text { Number of } \\
\text { patients }\end{array}$ & $\begin{array}{l}\text { Range dose } \\
\left(\mu \mathrm{g} \mathrm{kg}^{-1} \min ^{-1}\right)\end{array}$ \\
\hline \multicolumn{3}{|l|}{ Vasoactive medication } \\
\hline Dobutamine & 4 & $2-4$ \\
\hline Enoximone & 1 & \\
\hline Norepinephrine & 5 & $0.01-0.09$ \\
\hline Sodium nitroprusside & 1 & 0.25 \\
\hline
\end{tabular}

$C A B G$ Coronary artery bypass grafting, $A V R$ aortic valve replacement

Baseline Pmsf and Parm did not correlate with Pcv, Pa and pulse pressure. Baseline Pmsa correlated with Pcv (Pearson's correlation coefficient $R=0.846, p=0.001$ ) and with pulse pressure $(R=0.697, p=0.017)$. Pmsa did not correlate with mean, systolic and diastolic arterial pressure ( $p>0.28$ for all).

For the changes in Pmsf, Parm and Pmsa induced by HUT, only Pmsa correlated significantly with changes in $\operatorname{Pcv}(R=0.931, p<0.001)$. For the changes induced by VOL both Pmsf and Pmsa correlated with changes in Pcv $(R=0.781, p=0.005$ and $R=0.911, p<0.001)$. No significant correlation was found with changes in $\mathrm{Pa}$ or pulse pressure for changes in Pmsf, Parm and Pmsa.

Agreement of methods

For all measurements Pmsf and Parm displayed a nonsignificant bias of $-1.0 \pm 3.08 \mathrm{mmHg} \quad(p=0.062)$, COV of $15 \%$ and with LOA of -7.3 and $5.2 \mathrm{mmHg}$ (Fig. 2b). The biases for Pmsf and Parm were: baseline $-1.3 \pm 3.4$, HUT $-0.8 \pm 3.2$, VOL $-1.2 \pm 2.8 \mathrm{mmHg}$. For all measurements Pmsf and Pmsa displayed a bias of $-6.0 \pm 3.1 \mathrm{mmHg}(p<0.001), \mathrm{COV}$ of $17 \%$ and LOA of -12.4 and $0.3 \mathrm{mmHg}$ (Fig. 3b). The biases for Pmsf and Pmsa were: baseline $-5.0 \pm 2.8$, HUT $-5.3 \pm 3.2$ and VOL $-8.1 \pm 2.7 \mathrm{mmHg}$. Mean Pmsf, Parm and Pmsa across all three states were $20.9 \pm 5.6,19.8 \pm 5.7$ and $14.9 \pm 4.0 \mathrm{mmHg}$, respectively.

Changes of Parm $(\Delta$ Parm $)$ and Pmsa $(\Delta$ Pmsa) versus changes in Pmsf $(\Delta \mathrm{Pmsf})$ are shown in Fig. 4. Both $\Delta$ Parm and $\Delta$ Pmsa regressed significantly $(p<0.001)$ with $\Delta$ Pmsf (slope $=0.85, R=0.896$ and slope $=0.53$, $R=0.871$, respectively). The cross tabulation agreement of positive and negative changes in each of the methods for HUT and VOL displayed directionally balanced concordance for all data pairs for both $\Delta \mathrm{Parm}$ and $\Delta \mathrm{Pmsa}$ versus $\Delta$ Pmsf.

\section{Repeatability of Parm}

Bland-Altman analysis for Parm duplicate measurements during both baseline and VOL revealed a bias of $0.03 \pm 1.02 \mathrm{mmHg}$, LOA from -2.04 to $2.09 \mathrm{mmHg}$ and $\mathrm{COV}$ of $5 \%$. No difference was found between the

Table 3 Hemodynamic data of patients during baseline, HUT and VOL

\begin{tabular}{|c|c|c|c|c|c|c|c|c|}
\hline & \multicolumn{2}{|c|}{ Baseline } & \multicolumn{3}{|l|}{ HUT } & \multicolumn{3}{|l|}{ VOL } \\
\hline & Mean & SD & Mean & SD & $\mathrm{p} 1$ & Mean & SD & $\mathrm{p} 2$ \\
\hline $\mathrm{Pa}(\mathrm{mmHg})$ & 88.8 & 17.9 & 77.3 & 17.0 & $<0.001$ & 97.9 & 15.3 & 0.003 \\
\hline Psys (mmHg) & 128.5 & 21.9 & 107.2 & 16.9 & 0.001 & 143.3 & 17.7 & 0.004 \\
\hline Pdias (mmHg) & 69.0 & 17.7 & 62.4 & 17.9 & 0.001 & 75.2 & 15.6 & 0.040 \\
\hline $\mathrm{PP}(\mathrm{mmHg})$ & 59.5 & 14.7 & 44.8 & 9.9 & 0.016 & 68.1 & 12.1 & 0.076 \\
\hline $\mathrm{Pcv}(\mathrm{mmHg})$ & 7.1 & 2.0 & 4.4 & 1.8 & 0.001 & 10.4 & 1.3 & 0.001 \\
\hline $\mathrm{CO}\left(1 \min ^{-1}\right)$ & 5.8 & 1.6 & 4.8 & 1.2 & 0.006 & 7.0 & 1.7 & 0.004 \\
\hline $\operatorname{HR}\left(\min ^{-1}\right)$ & 88 & 14 & 87 & 15 & 0.574 & 86 & 10 & 0.475 \\
\hline Pmsf (mmHg) & 19.7 & 3.9 & 16.2 & 3.0 & 0.001 & 28.3 & 3.6 & $<0.001$ \\
\hline Parm (mmHg) & 18.4 & 3.7 & 15.4 & 3.1 & 0.001 & 27.1 & 4.0 & $<0.001$ \\
\hline Pmsa (mmHg) & 14.7 & 2.7 & 10.9 & 2.0 & $<0.001$ & 19.2 & 1.1 & $<0.001$ \\
\hline
\end{tabular}

Values are mean $\pm \mathrm{SD} ; n=11$ patients

HUT Head-up tilt, VOL after volume loading (+500 ml), $P a$ arterial pressure, $P$ sys systolic arterial pressure, Pdias diastolic arterial pressure, $P P$ pulse pressure, $P c v$ central venous pressure, $C O$ cardiac output, $H R$ heart rate, $P m s f$ mean systemic filling pressure,
Parm arm equilibrium pressure, Pmsa model analog mean circulatory pressure

Statistical comparison, $\mathrm{p} 1$ and $\mathrm{p} 2$, paired $t$ test between baseline and HUT and between baseline and VOL 


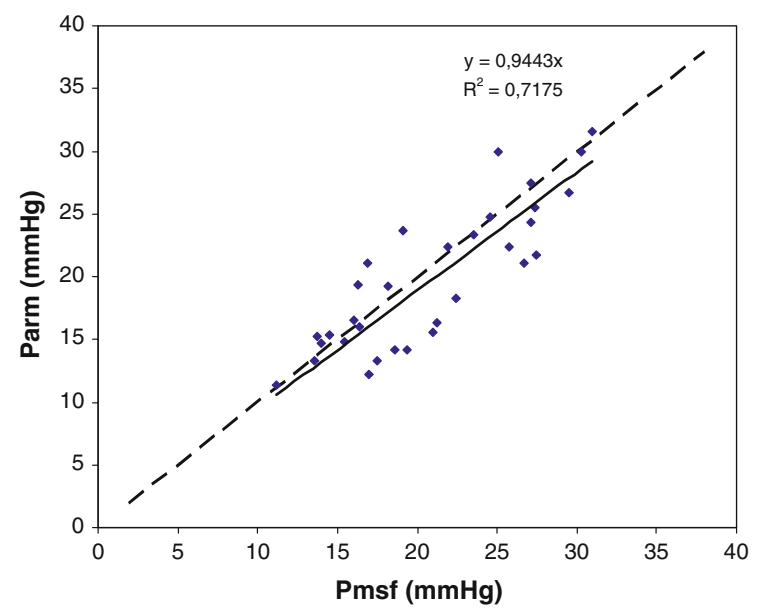

Fig. 2 Regression (a) and Bland-Altman analysis (b) of arm equilibrium pressure (Parm) and mean systemic filling pressure (Pmsf). In a, the solid line is the regression line, and the dashed line

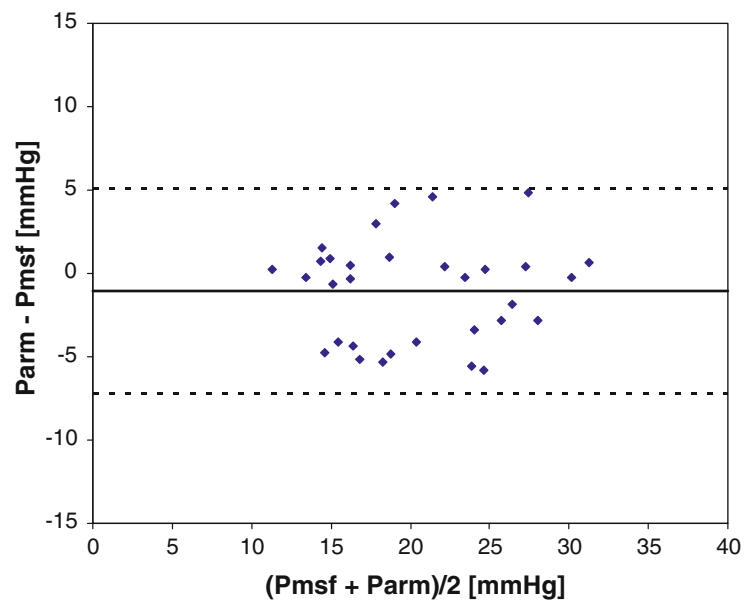

is the line of identity. In $\mathbf{b}$, the solid line indicates the bias, and the dashed lines are the limits of agreement

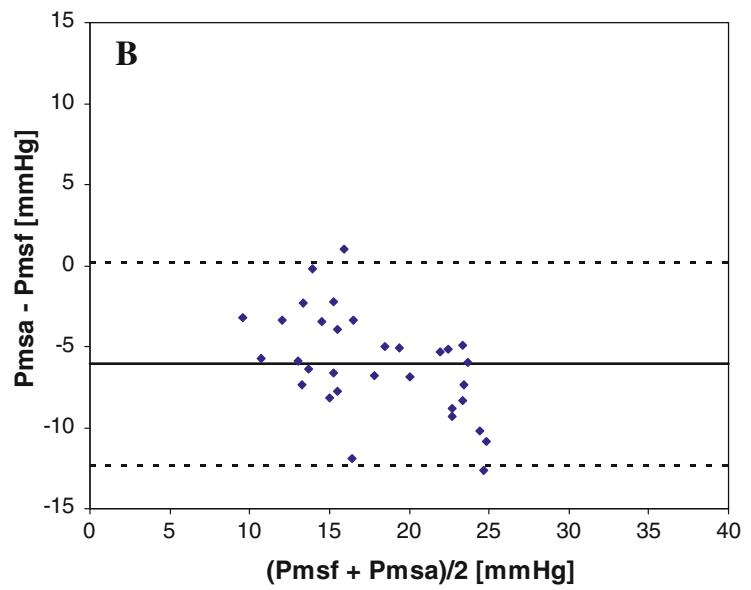

line of identity. In $\mathbf{b}$, the solid line indicates the bias, and the dashed lines are the limits of agreement

state flow conditions the arm is representative of the entire circulation, such that a rapid vascular occlusion will result in its stop-flow $\mathrm{Pa}$ approximating Pmsf. Thus, both Pmsf and Parm can be used at the bedside to measure effective circulating blood volume. Furthermore, Pmsa can reliably tract changes in effective circulating blood volume status.

The use of both Parm and Pmsa has practical advantages over our previously validated inspiratory-hold maneuver Pmsf approach. Neither requires positivepressure breathing or multiple simultaneous measures of Pcv and CO during inspiratory hold maneuvers, and both can be rapidly and repeatedly measured sequentially as treatment or time progresses. Parm requires only the peripheral arterial catheter. Pmsa requires both central previously validated, by Anderson [8], that during steady-

Pon aflow (Parm) are interchangea Pmsf calculated using inspiratory hold maneuvers in mechanically ventilated postoperative cardiac surgery patients. Furthermore, changes in volume status by HUT and VOL are similarly tracked by Pmsf, Parm and Pmsa.
These data support the hypothesis formulated, but not 

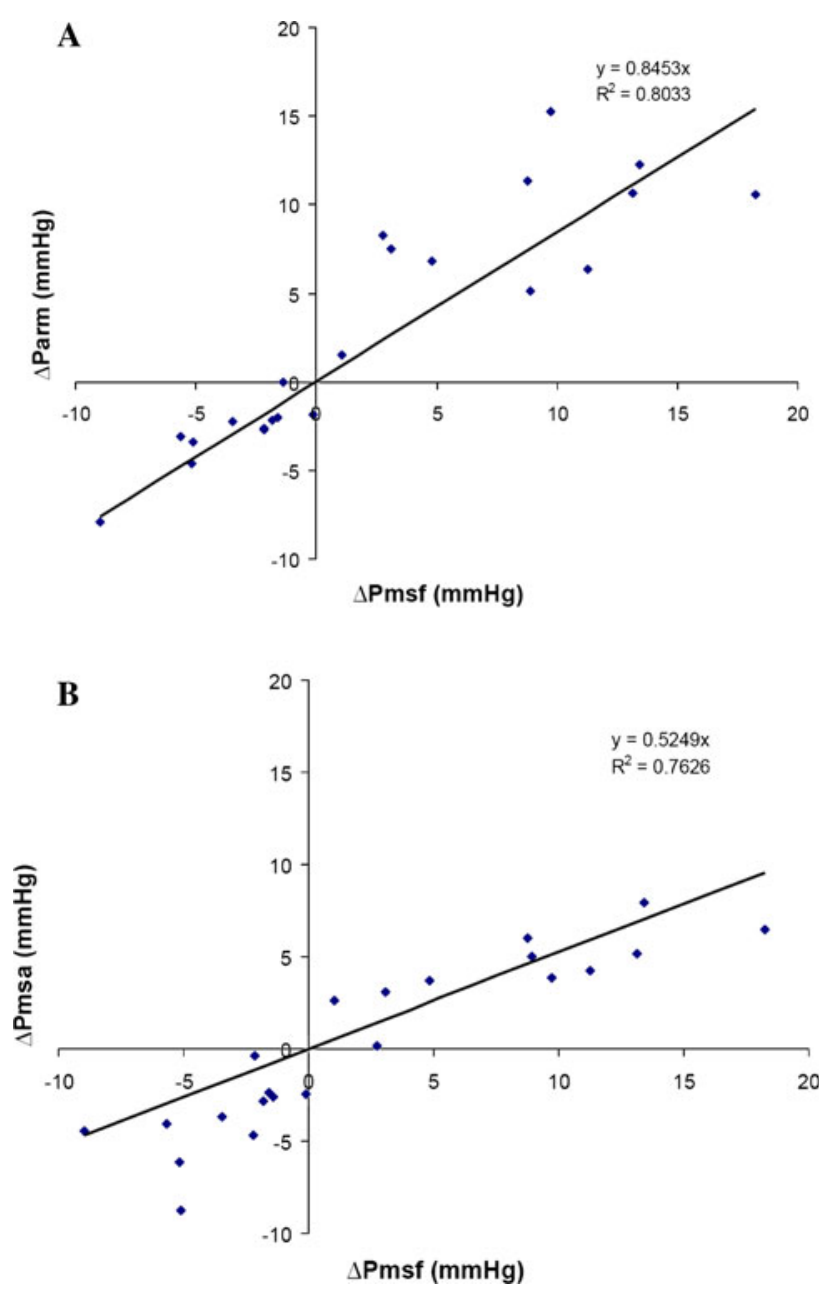

Fig. 4 Changes in mean systemic filling pressure by arm equilibrium pressure $(\Delta$ Parm $)($ a) and by model analog $(\Delta$ Pmsa) (b) plotted against changes in mean systemic filling pressure by inspiratory hold procedures ( $\Delta \mathrm{Pmsf})$. The regression line is indicated by a solid line

venous and peripheral arterial catheters. Thus, these two novel approaches markedly increase the applicability of assessment of effective circulating blood volume to a broader patient population.

\section{Methodological considerations}

\section{Radial artery pressure}

Shortly after cardiopulmonary bypass, radial artery pressure can be significantly less than aortic pressure [13-15], but this difference disappears after about $60 \mathrm{~min}$, coinciding with hemodynamic stabilization [13]. Our study started after approximately $2 \mathrm{~h}$ after cardiopulmonary bypass in stable patients. Therefore, we believe that the mean radial artery pressure reliably reflected the central aortic pressure. We recently documented in a porcine model of acute endotoxemia [16] that similar central-toregional arterial pulse pressure changes occur. However, the value of Pmsf is not dependent on the calibration of the pulse contour method as long as a linear change in $\mathrm{CO}$ is followed by a linear change in $\mathrm{CO}$ derived from the pulse contour. Indeed, Pcv at CO equal to zero is not even influenced by a calibration factor.

\section{Arm stop-flow procedure}

In the pilot stop-flow study described above, we observed that a plateau pressure developed in both arterial and venous pressures after 20-30 s, as predicted by Anderson [8]. However, a further decrement in both $\mathrm{Pa}$ and $\mathrm{Pv}$ developed after 35-40 s, indicating probable hypoxiainduced vasodilation. We also observed the best repeatability and lowest standard deviations between the arterial and venous pressure at 25-30 s of stop-flow, which was the time we used in this study. Furthermore, stop-flow durations longer than $5 \mathrm{~min}$ are needed to produce reactive hyperemia in the human forearm [17, 18]. Thus, if stop-flow maneuvers are limited to $<1 \mathrm{~min}$, regional blood flow will also normalize after an additional $1 \mathrm{~min}$ [19]. The rapid cuff inflator inflates in less than $0.3 \mathrm{~s} \mathrm{[20].}$ In this time there is only a brief cessation of venous return prior to arterial stop-flow equal to approximately one heart beat. We expect this overestimation to be negligible because the amount of inflow is small compared to the total distal arm blood volume. Finally, since longer vascular occlusion maneuvers are routinely used to assess dynamic changes in tissue $\mathrm{O}_{2}$ saturation without complications [21], we feel that this much shorter vascular occlusion maneuver is safe.

\section{Model analog Pmsa}

No clinical evaluation of Pmsa against other methods to measure Pmsf has been done so far. The validity of the Pmsa algorithm was successfully tested using a closed loop control of fluid replacement during continuous hemodiafiltration [22]. Our data support these findings because $\Delta$ Pmsf and $\Delta$ Pmsa faithfully track each other.

\section{Pmsf}

We showed that Modelflow pulse contour CO was interchangeable with pulmonary artery and aortic flow probederived CO in swine [23], and that Modelflow-derived Pmsf was interchangeable with flow probe-derived Pmsf with a COV for duplicate measurements of $6.1 \%$. Still, we report mean baseline Pmsf values of $19.7 \mathrm{mmHg}$ in our cardiac surgical patients, which are higher than the Pmsf values reported between 7 and $12 \mathrm{mmHg}$ in animal 
studies [24-27]. Using the same inspiratory hold technique and pulse contour analysis, we found Pmsf values of $10.38 \pm 1.09 \mathrm{mmHg}$ in pigs [23]. A primary difference between the prior animal studies and our patient observations is the difference in baseline Pcv. In the animal studies this value is close to zero, whereas Pcv in our patient population is $7.1 \mathrm{mmHg}$ on average. The pressure gradient for venous return (Pmsf minus Pcv) in our study $(12-13 \mathrm{mmHg})$ is therefore similar to the pressure gradient for venous return in the animal studies. Thus, our Pmsf values are coupled with the increased Pcv. However, high Pmsf values still predispose patients to peripheral edema formation.

Jellinek et al. [28] and Schipke et al. [29] estimated Pmsf in patients during episodes of apnea and ventricular fibrillation, and found a mean Pmsf value of 10.2 and $12 \mathrm{mmHg}$, respectively. However, both studies were done on highly anesthetized, non-volume resuscitated subjects. Our method for estimating Pmsf differs considerably from stopping flow by ventricular fibrillation, and our method allows an estimation of Pmsf with intact circulation, applicable in the intensive care unit $[5,30]$.

\section{Agreement among Parm, Pmsa and Pmsf}

We found agreement among Pmsf and Parm (Fig. 2), and $\Delta$ Pmsf and $\Delta$ Parm were concordant in all interventions (Fig. 4). Therefore, both methods should equally measure and follow changes in effective circulating blood volume. There was poor agreement between Pmsa and Pmsf. The large bias makes the methods non-interchangeable. However, the full concordance between $\Delta$ Pmsf and $\Delta$ Pmsa indicates that the Pmsa method is very applicable to track changes in effective circulating blood volume, as indeed was documented by Parkin et al. [22] in dialysis patients.

Finally, effective circulating blood volume is a functional measure, not an absolute one. In our study the vasoactive medication was not changed. Changing vasomotor tone will alter unstressed volume, stressed volume and compliance. Any treatment that alters unstressed volume will also alter effective circulating blood volume independent of changes in blood volume, as was demonstrated by Guyton et al. [4].

Can either Parm or Pmsa replace the Pmsf method in the bedside assessment of effective circulating blood volume? Based on the established argument of Critchley and Critchley [31], a new method may replace an older established method if the new method itself has errors not greater than the older method. The Parm method showed a non-significant bias when compared to Pmsf. A single measurement of Pmsf has a COV of about $6 \%$ [23]. We found a $5 \%$ repeatability for Parm. Thus, our data support the argument that Parm may replace inspiratory holdmaneuver generated Pmsf.
A significant bias $(p<0.001)$ was observed between Pmsf and Pmsa, precluding the substitution of raw Pmsa values for Pmsf. However, based on the linearity of Pmsf and Pmsa (Fig. 3a) one can adjust the Pmsa values using a calibration factor of 1.42 (i.e., the reciprocal of the slope of the regression 0.704). After this calibration is applied to Pmsa values, indicated in Fig. 3a by an arrow from the regression line to the line of identity, the bias reduces to zero. The expected precision of the calculation of Pmsa is approximately $10 \%$ (this COV is largely caused by the COV in Pcv measurement; a value of $10 \mathrm{mmHg}$ can be 9.51 or $10.49 \mathrm{mmHg}$ ). Although this $10 \%$ is higher than the $6 \%$ for Pmsf, after recalibration, the Pmsa model analog may replace Pmsf. It must be emphasized that the correction factor only describes our postoperative cardiac surgery population and will require similar validation in other patient groups.

\section{Study limitations}

The number of patients included in the study is relatively low. However, we still found a significant difference between Pmsa and Pmsf. With a larger study population the difference between Pmsf and Parm could have become significant. However, the absolute difference of $-1.0 \mathrm{mmHg}$ is not clinically relevant. We included patients with preserved left ventricular function after relatively simple cardiac surgery, and excluded patients with previous myocardial infarction and/or congestive heart failure (New York Heart Association class 4). These patients are known to have markedly increased vascular tone with an associated decreased proportional unstressed vascular volume. Thus, caution needs to be used when extrapolating the accuracy of these comparisons to other patients groups. During the study we made no changes in medication. Therefore, we cannot report on the values and comparison of Pmsf, Parm and Pmsa during changes in vasoactive medication, which influences vascular elastance, resistance and conductance properties. A fundamental limitation of the Parm technique is the need to measure arterial pressure from a radial arterial site. In patients with sepsis or on high levels of vasopressors, radial artery compliance may not reflect central arterial compliance, although the mean Pa remains accurate [16]. Therefore, in these patients it is not clear if Parm or Pmsa will tract Pmsf. Still, under these conditions, the diagnosis of decreased effective circulating blood volume is rarely an issue.

\section{Conclusions}

The equilibrium pressure in the arm during stop-flow (Parm) and inspiratory-hold maneuver-derived Pmsf 
values are interchangeable in mechanically ventilated postoperative cardiac surgery patients. Thus, the mean systemic filling pressure can be simply measured at the bedside by measuring arterial pressure during upper arm stop-flow, without the need for inspiratory hold maneuvers or central venous or pulmonary artery catheters. Furthermore, changes in effective circulatory volume are accurately trended by changes in both Parm and Pmsa.
Acknowledgments The study was performed in Leiden supported by institutional grants. M. Pinsky was supported by NIH grant HL67181.

Open Access This article is distributed under the terms of the Creative Commons Attribution License which permits any use, distribution, and reproduction in any medium, provided the original author(s) and the source are credited.

\section{References}

1. Wiedemann HP, Wheeler AP, Bernard GR, Thompson BT, Hayden D, deBoisblanc B, Connors AF Jr, Hite RD, Harabin AL (2006) Comparison of two fluid-management strategies in acute lung injury. N Engl J Med 354:2564-2575

2. Huang SJ, Hong WC, Han YY, Chen YS, Wen CS, Tsai YS, Tu YK (2006) Clinical outcome of severe head injury using three different ICP and CPP protocol-driven therapies. J Clin Neurosci 13:818-822

3. Brandstrup B, Tonnesen H, BeierHolgersen R, Hjortso E, Ording $\mathrm{H}$, Lindorff-Larsen K, Rasmussen MS, Lanng C, Wallin L, Iversen LH, Gramkow CS, Okholm M, Blemmer T, Svendsen PE, Rottensten HH, Thage B, Riis J, Jeppesen IS, Teilum D, Christensen AM, Graungaard B, Pott F (2003) Effects of intravenous fluid restriction on postoperative complications: comparison of two perioperative fluid regimens: a randomized assessor-blinded multicenter trial. Ann Surg 238:641-648

4. Guyton AC, Polizo D, Armstrong GG (1954) Mean circulatory filling pressure measured immediately after cessation of heart pumping. Am J Physiol 179:261-267

5. Maas JJ, Geerts BF, van den Berg PC, Pinsky MR, Jansen JR (2009) Assessment of venous return curve and mean systemic filling pressure in postoperative cardiac surgery patients. Crit Care Med 37:912-918

6. Jansen JR, Maas JJ, Pinsky MR (2010) Bedside assessment of mean systemic filling pressure. Curr Opin Crit Care 16:231-236

7. Geerts BF, Maas J, de Wilde RB, Aarts LP, Jansen JR (2011) Arm occlusion pressure is a useful predictor of an increase in cardiac output after fluid loading following cardiac surgery. Eur J Anaesthesiol 28:802-806

8. Anderson RM (1993) The gross physiology of the cardiovascular system. Racquet Press, Tucson
9. Parkin WG, Leaning MS (1993) Therapeutic control of the circulation. J Clin Monit Comput 22:391-400

10. Wesseling KH, Jansen JR, Settels JJ, Schreuder JJ (1993) Computation of aortic flow from pressure in humans using a nonlinear, three-element model. J Appl Physiol 74:2566-2573

11. Jansen JR, Schreuder JJ, Mulier JP, Smith NT, Settels JJ, Wesseling KH (2001) A comparison of cardiac output derived from the arterial pressure wave against thermodilution in cardiac surgery patients. Br J Anaesth $87: 212-222$

12. de Wilde RB, Schreuder JJ, van den Berg PC, Jansen JR (2007) An evaluation of cardiac output by five arterial pulse contour techniques during cardiac surgery. Anaesthesia 62:760-768

13. Stern DH, Gerson JI, Allen FB, Parker FB (1985) Can we trust the direct radial artery pressure immediately following cardiopulmonary bypass? Anesthesiology 62:557-561

14. Pauca AL, Hudspeth AS, Wallenhaupt SL, Tucker WY, Kon ND, Mills SA, Cordell AR (1989) Radial artery-toaorta pressure difference after discontinuation of cardiopulmonary bypass. Anesthesiology 70:935-941

15. Rich GF, Lubanski RE Jr, McLoughlin TM (1992) Differences between aortic and radial artery pressure associated with cardiopulmonary bypass. Anesthesiology 77:63-66

16. Hatib F, Jansen JR, Pinsky MR (2011) Peripheral vascular decoupling in porcine endotoxic shock. J Appl Physiol 111:853-860

17. Betik AC, Luckham VB, Hughson RL (2004) Flow-mediated dilation in human brachial artery after different circulatory occlusion conditions. Am J Physiol Heart Circ Physiol 286:H442H448
18. Katz SD, Rao R, Berman JW, Schwarz M, Demopoulos L, Bijou R, LeJemtel TH (1994) Pathophysiological correlates of increased serum tumor necrosis factor in patients with congestive heart failure. Relation to nitric oxide-dependent vasodilation in the forearm circulation. Circulation 90:12-16

19. Sumner DS (1985) Volume plethysmography in vascular disease: an overview. In: Berstein EF (ed) Noninvasive diagnostic techniques in vascular disease, 3rd edn. CV Mosby, St. Louis, pp 97-118

20. Chu AC, St Andrew D (1983) Efficient, inexpensive rapid cuff inflator for venous occlusion plethysmography. Clin Phys Physiol Meas 4:339-341

21. Gomez H, Torres A, Polanco P, Kim HK, Zenker S, Puyana JC, Pinsky MR (2008) Use of non-invasive NIRS during a vascular occlusion test to assess dynamic tissue $\mathrm{O}(2)$ saturation response. Intensive Care Med 34:1600-1607

22. Parkin G, Wright C, Bellomo R, Boyce $N$ (1994) Use of a mean systemic filling pressure analogue during the closedloop control of fluid replacement in continuous hemodiafiltration. J Crit Care 9:124-133

23. Maas JJ, Geerts BF, Jansen JR (2011) Evaluation of mean systemic filling pressure from pulse contour cardiac output and central venous pressure. J Clin Monit Comput 25:193-201

24. Guyton AC, Lindsey AW, Abernathy B, Richardson T (1957) Venous return at various right atrial pressures and the normal venous return curve. Am J Physiol 189:609-615

25. Pinsky MR (1984) Instantaneous venous return curves in an intact canine preparation. J Appl Physiol 56:765-771

26. Greene AS, Shoukas AA (1986) Changes in canine cardiac function and venous return curves by the carotid baroreflex. Am J Physiol 251:H288H296 
27. Versprille A, Jansen JR (1985) Mean systemic filling pressure as a characteristic pressure for venous return. Pflugers Arch 405:226-233

28. Jellinek H, Krenn H, Oczenski W, Veit F, Schwarz S, Fitzgerald RD (2000) Influence of positive airway pressure on the pressure gradient for venous return in humans. J Appl Physiol 88:926-932
29. Schipke JD, Heusch G, Sanii AP, Gams E, Winter J (2003) Static filling pressure in patients during induced ventricular fibrillation. Am J Physiol Heart Circ Phys 285:H2510-H2515

30. Maas JJ, de Wilde RB, Aarts LP, Pinsky MR, Jansen JR (2012) Determination of vascular waterfall phenomenon by bedside measurement of mean systemic filling pressure and critical closing pressure in the intensive care unit. Anesth Analg 114:803-810
31. Critchley LA, Critchley JA (1999) A meta-analysis of studies using bias and precision statistics to compare cardiac output measurement techniques. J Clin Monit Comput 15:85-91 